\title{
STABLE ATTRACTING SETS IN DYNAMICAL SYSTEMS AND IN THEIR ONE-STEP DISCRETIZATIONS*
}

\author{
P. E. KLOEDEN†‡ AND J. LORENZ $\dagger$
}

\author{
Dedicated to Herbert B. Keller on the occasion of his 60 th birthday
}

\begin{abstract}
We consider a dynamical system described by a system of ordinary differential equations which possesses a compact attracting set $\Lambda$ of arbitrary shape. Under the assumption of uniform asymptotic stability of $\Lambda$ in the sense of Lyapunov, we show that discretized versions of the dynamical system involving one-step numerical methods have nearby attracting sets $\Lambda(h)$, which are also uniformly asymptotically stable. Our proof uses the properties of a Lyapunov function which characterizes the stability of $\Lambda$.
\end{abstract}

Key words. attracting set, dynamical system, discretization, Lyapunov function

AMS(MOS) subject classifications. 65L05, 34C35

1. Introduction. Much of what we know about chaotic behavior in specific continuous time dynamical systems, such as the Lorenz equations, has been suggested by numerical studies [6], [8]. An underlying belief here is that the basic qualitative features of the dynamical systems are not significantly changed by the discretization process associated with the numerical method. Standard convergence results for a numerical method are, however, typically only applied to individual trajectories and give error bounds of the form $e^{c T} h^{p}$ where $h$ is the step-size, $p$ is the order of the method and $T$ is the length of the time interval under consideration. For large $T$ such estimates become useless. In view of this, what can numerical results tell us about the longtime behavior of dynamical systems?

Longtime comparisons are possible in the simple cases where the continuous time system has an asymptotically stable steady or periodic solution. Various results and techniques are discussed, for example, in [1]-[5], [9]. For more complicated attracting sets little appears known. Indeed, the highly sensitive dependence on initial conditions within a strange attractor suggests that there is little prospect of closely following a given trajectory within such a set with a numerical method. Nevertheless, it is possible, as we shall show here, to obtain an attracting set for the discretized system which is close to that of the original continuous time system. Our result is independent of the geometrical shape of the attracting set. We shall not, however, say anything here about the comparative dynamics within these asymptotically stable attracting sets. Our choice of the terminology "attracting set" rather than "attractor" reflects this omission, with the latter term being reserved to mean an attracting set which contains a dense trajectory (so $\{0\}$ is an attractor for the simple system $d x / d t=-x$, whereas any set $[-\varepsilon, \varepsilon]$ is an attracting set).

We consider an autonomous system of ordinary differential equations

$$
\frac{d x}{d t}=F(x)
$$

\footnotetext{
* Received by the editors May 28, 1985, and in revised form January 20, 1986. This research was supported by National Science Foundation Grants DMS83-12264 and DMS84-00885, and by U.S. Army contract DAAG29-85-K-0092.

$\dagger$ Department of Applied Mathematics, California Institute of Technology, Pasadena, California 91125.

$\ddagger$ Permanent address, School of Mathematical and Physical Sciences, Murdoch University, Murdoch 6150, Western Australia.
} 
in $\mathfrak{R}^{N}$ for any $N \geqq 1$ and discrete analogues described by one-step numerical methods of $p$ th order $(p \geqq 1)$

$$
x_{n+1}=x_{n}+h F_{h}\left(x_{n}\right)
$$

in $\mathfrak{R}^{N}$ with uniform step-size $h$. Our main result is

THEOREM 1.1. Suppose that $F$ and its first $p$ derivatives are uniformly bounded in $\mathfrak{R}^{N}$ and that 1.1 has a compact, uniformly asymptotically stable set $\Lambda$.

Then there is $h_{2}>0$ such that for each $0<h<h_{2}(1.2)$ has a compact, uniformly asymptotically stable set $\Lambda(h)$ which contains $\Lambda$ and converges to $\Lambda$ with respect to the Hausdorff metric as $h \rightarrow 0+$. Moreover, there is a bounded, open set $U_{0}$, which is independent of $h$ and contains $\Lambda(h)$, and a time

$$
T_{0}(h)=A+B p \log \frac{1}{h},
$$

where $A$ and $B$ are constants depending on the stability characteristics of $\Lambda$, such that the iterates of (1.2) satisfy

$$
x_{n} \in \Lambda(h)
$$

for all $n h \geqq T_{0}(h), x_{0} \in U_{0}$ and $0<h<h_{2}$.

In the following sections we shall give definitions of the uniform asymptotic stability of a set and the Hausdorff metric on nonempty, compact subsets of $\Re^{N}$. We mean stability in the sense of Lyapunov and shall use Lyapunov functions to characterize the stability of a set. These concepts are introduced in $\S 2$ and our proof of Theorem 1.1 is presented in $\S 3$ as a sequence of lemmata. Finally in $\S 4$ we discuss various consequences and generalizations of this theorem. In particular we shall see that the sets $\Lambda(h)$ are usually not minimal attractors, that is they do not contain a dense trajectory for the discretized systems (1.2).

2. Asymptotically stable attracting sets. We consider compact sets $\Lambda$ of unspecified shape which are positively invariant and uniformly asymptotically stable with respect to a given autonomous system of differential equations

$$
\frac{d x}{d t}=F(x)
$$

in $\mathfrak{R}^{N}$. As well as the familiar singleton sets (steady solutions), these sets $\Lambda$ include closed curves (periodic solutions), toroidal and stranger shaped sets, and also sets containing more than one distinct trajectory of (2.1). We shall assume for simplicity that all of the solutions of (2.1) are ultimately contained in some possibly large, bounded subset of $\mathfrak{R}^{N}$, hence exist for all future time, and that $F$ is uniformly Lipschitzian on this subset. (Stronger smoothness assumptions of $F$ will be appropriate later when numerical methods are discussed.)

Let $\Lambda$ be a nonempty, compact subset of $\mathfrak{R}^{N}$ and let $x \in \mathfrak{R}^{N}$. Then

$$
\operatorname{dist}(x, \Lambda)=\inf \{|x-\bar{x}| ; \bar{x} \in \Lambda\},
$$

where the infimum is actually attained because $\Lambda$ is nonempty and compact. Following Yoshizawa $[10, \S 16]$ we say that $\Lambda$ is uniformly stable for (2.1) if for each $\varepsilon>0$ there exists a $\delta=\delta(\varepsilon)>0$ such that

$$
\operatorname{dist}\left(x\left(t ; x_{0}\right), \Lambda\right)<\varepsilon
$$

for all $t \geqq 0$ whenever dist $\left(x_{0}, \Lambda\right)<\delta$, where $x\left(t ; x_{0}\right)$ is the solution of (2.1) with initial value $x\left(0 ; x_{0}\right)=x_{0}$. The set $\Lambda$ is then positively invariant for $(2.1)$, that is $x\left(t ; x_{0}\right) \in \Lambda$ 
for all $t \geqq 0$ and all $x_{0} \in \Lambda$. If in addition there exists a $\delta_{0}>0$ and for each $\varepsilon>0$ a time $T(\varepsilon)>0$ such that

$$
\operatorname{dist}\left(x\left(t ; x_{0}\right), \Lambda\right)<\varepsilon \quad \text { for all } t \geqq T(\varepsilon)
$$

whenever dist $\left(x_{0}, \Lambda\right)<\delta_{0}$, we say that $\Lambda$ is uniformly asymptotically stable for (2.1).

Lyapunov functions may be used to characterize the stability of an arbitrarily shaped $\Lambda$ for which there is no simple spectral theory as when $\Lambda$ is a singleton set. In simple mechanical systems they represent the potential energy, which decreases along trajectories of the system. Unlike linearized spectral theory, the use of Lyapunov functions is not just local. Moreover it does not require explicit knowledge of the solutions of the differential equation. Yoshizawa [10] gives various necessary conditions and sufficient conditions involving the existence of Lyapunov functions for a compact set $\Lambda$ to be uniformly asymptotically stable for a differential equation (2.1). See also [9]. The following theorem of necessary conditions is a restatement of Theorem 22.5 in [10]. These conditions are also sufficient, as can be deduced from $\S \S 14$ and 16 of [10], but we shall not require that here. We define

$$
S\left(\Lambda ; R_{0}\right)=\left\{x \in \mathfrak{R}^{N} ; \operatorname{dist}(x, \Lambda)<R_{0}\right\} .
$$

THEOREM 2.1. Suppose that the nonempty, compact subset $\Lambda$ of $\mathfrak{R}^{N}$ is uniformly asymptotically stable for (2.1) and that $F$ is uniformly Lipschitzian on some sufficiently large neighborhood of $\Lambda$. Then there exists a function

$$
V: S\left(\Lambda ; R_{0}\right) \rightarrow[0, \infty)
$$

for some $R_{0}>0$ for which:

(i) $V$ is uniformly Lipschitzian on $S\left(\Lambda ; R_{0}\right)$, i.e. there exists a constant $L>0$ such that

$$
\left|V(x)-V\left(x^{\prime}\right)\right| \leqq L\left|x-x^{\prime}\right|
$$

for all $x, x^{\prime} \in S\left(\Lambda ; R_{0}\right)$;

(ii) there exist continuous strictly increasing functions $\alpha, \beta:\left[0, R_{0}\right) \rightarrow[0, \infty)$ with $\alpha(0)=\beta(0)=0$ and $\alpha(r)<\beta(r)$ for $r>0$ such that

$$
\alpha(\operatorname{dist}(x, \Lambda)) \leqq V(x) \leqq \beta(\operatorname{dist}(x, \Lambda))
$$

for all $x \in S\left(\Lambda ; R_{0}\right)$; and

(iii) there exists a constant $c>0$ such that

$$
D_{(2.1)}^{+} V(x) \leqq-c V(x)
$$

for all $x \in S\left(\Lambda ; R_{0}\right)$, where the upper-Dini derivative of $V$ with respect to (2.1) is defined as

$$
D_{(2.1)}^{+} V(x)=\varlimsup_{h \downarrow 0}\{V(x+h F(x))-V(x)\} / h^{1}
$$

This theorem guarantees the existence of such a Lyapunov function, but gives no practical information on how to find it. Nevertheless for such a function $V$ it follows from (ii) and (iii) that $x\left(t ; x_{0}\right) \in S\left(\Lambda ; R_{0}\right)$ and

$$
V\left(x\left(t ; x_{0}\right)\right) \leqq e^{-c t} V\left(x_{0}\right)
$$

\footnotetext{
${ }^{1}$ This equals $\nabla V(x) \cdot F(x)$ when $V$ is differentiable.
} 
for all $t \geqq 0$ whenever dist $\left(x_{0}, \Lambda\right)<R_{1}<R_{0}$, where $R_{1}$ is defined by $\beta\left(R_{1}\right)=\alpha\left(R_{0}\right)$. To see this, suppose that there is a $0<T<\infty$ such that

$$
\operatorname{dist}\left(x\left(t ; x_{0}\right), \Lambda\right)<R_{0} \quad \text { for } 0 \leqq t<T,
$$

but

$$
\operatorname{dist}\left(x\left(T ; x_{0}\right), \Lambda\right)=R_{0} .
$$

It follows from (iii) that the inequality (2.2) certainly holds over $0 \leqq t<T$, and so by continuity also for $t=T$, that is

$$
V\left(x\left(T ; x_{0}\right)\right) \leqq e^{-c T} V\left(x_{0}\right) .
$$

Then from (ii)

$$
\begin{aligned}
\alpha\left(R_{0}\right) & =\alpha\left(\operatorname{dist}\left(x\left(T ; x_{0}\right), \Lambda\right)\right) \\
& \leqq V\left(x\left(T ; x_{0}\right)\right) \\
& \leqq e^{-c T} V\left(x_{0}\right) \\
& \leqq e^{-c T} \beta\left(\operatorname{dist}\left(x_{0}, \Lambda\right)\right) \\
& <e^{-c T} \beta\left(R_{1}\right)=e^{-c T} \alpha\left(R_{0}\right),
\end{aligned}
$$

which is a contradiction, so no such $T$ exists.

3. Proof of main result. For simplicity of presentation we suppose that the function $F$ in the differential equation $(2.1)$ is $p \geqq 1$ times continuously differentiable and that these derivatives are globally bounded in $\mathfrak{R}^{N}$. Then all solutions $x\left(t ; x_{0}\right)$ of $(2.1)$ exist for all future time and their first $p+1$ time derivatives are globally and uniformly bounded. Thus it is a reasonable assumption for a one-step method (1.2) that there exists a constant $C_{p}$, which is independent of $h$ and $x_{0}$, for which we have a local estimate

$$
\left|x_{1}(h)-x\left(h ; x_{0}\right)\right| \leqq C_{p} h^{p+1} .
$$

Here $x_{1}(h)=x_{1}=x_{0}+h F_{h}\left(x_{0}\right)$ according to (1.2). (We sometimes write the iterates as functions of $h$ to emphasize the dependency.) Furthermore, let $L_{p}(h)$ be a global Lipschitz constant for $F_{h}(x)$.

We assume that the differential system (2.1) has a compact, uniformly asymptotically stable set $\Lambda$ and that $V: S\left(\Lambda ; R_{0}\right) \rightarrow[0, \infty)$ is an appropriate Lyapunov function, the existence of which is guaranteed by Theorem 2.1 , with constants $c, L, R_{0}, R_{1}$ and functions $\alpha, \beta$. We shall construct a compact subset $\Lambda(h)$ which contains $\Lambda$ in its interior and which is uniformly asymptotically stable for the discrete dynamical system (1.2) with step-size $h$ (under a definition analogous to that in the previous section). For clarity our construction and proof is presented as a sequence of lemmata.

LeMMA 3.1. There exist $0<r_{0}<R_{1}$ and $h_{0}>0$ such that

$$
x_{1}(h) \in S\left(\Lambda ; R_{0}\right)
$$

whenever $x_{0} \in S\left(\Lambda ; r_{0}\right)$ and $h \leqq h_{0}$.

Proof. We define $r_{0}$ by $\beta\left(r_{0}\right)=\alpha\left(R_{0} / 2\right)$ and $h_{0}$ by $C_{p} h_{0}^{p+1}=R_{0} / 2$. Then $r_{0}<R_{1}<R_{0}$, so for any $x_{0} \in S\left(\Lambda ; r_{0}\right)$ we have $x\left(t ; x_{0}\right) \in S\left(\Lambda ; R_{0}\right)$ for all $t>0$ and inequality (2.2) is satisfied. In particular for $t=h$

$$
V\left(x\left(h ; x_{0}\right)\right) \leqq e^{-c h} V\left(x_{0}\right)<V\left(x_{0}\right) .
$$

By condition (ii) of Theorem 2.1

$$
\alpha\left(\operatorname{dist}\left(x\left(h ; x_{0}\right), \Lambda\right)\right) \leqq V\left(x\left(h ; x_{0}\right)\right)
$$


and

$$
V\left(x_{0}\right) \leqq \beta\left(\operatorname{dist}\left(x_{0}, \Lambda\right)\right)<\beta\left(r_{0}\right)=\alpha\left(\frac{R_{0}}{2}\right) .
$$

In view of (3.2) we thus have

$$
\operatorname{dist}\left(x\left(h ; x_{0}\right), \Lambda\right)<\frac{R_{0}}{2},
$$

which we combine with (3.1) and the definition of $h_{0}$ to get

$$
\begin{aligned}
\operatorname{dist}\left(x_{1}(h), \Lambda\right) & \leqq\left|x_{1}(h)-x\left(h ; x_{0}\right)\right|+\operatorname{dist}\left(x\left(h ; x_{0}\right), \Lambda\right) \\
& <C_{p} \cdot h_{0}^{p+1}+\frac{R_{0}}{2} \\
& \leqq R_{0},
\end{aligned}
$$

as long as $h \leqq h_{0}$.

As a consequence of Lemma 3.1 both $x\left(h ; x_{0}\right)$ and $x_{1}(h)$ belong to $S\left(\Lambda ; R_{0}\right)$, the domain of definition of the Lyapunov function $V$, whenever $x_{0} \in S\left(\Lambda ; r_{0}\right)$ and $h \leqq h_{0}$. Then by the Lipschitz property of $V$ and inequality (3.1)

$$
\begin{aligned}
\left|V\left(x_{1}(h)\right)-V\left(x\left(h ; x_{0}\right)\right)\right| & \leqq L\left|x_{1}(h)-x\left(h ; x_{0}\right)\right| \\
& \leqq L C_{p} h^{p+1},
\end{aligned}
$$

which combines with inequality (3.2) to give

$$
V\left(x_{1}(h)\right) \leqq e^{-c h} V\left(x_{0}\right)+L C_{p} h^{p+1} .
$$

LEMMA 3.2. The open set

$$
U_{0}=\left\{x \in S\left(\Lambda ; R_{0}\right) ; V(x)<\alpha\left(r_{0}\right)\right\}
$$

satisfies $\Lambda \subset U_{0} \subset S\left(\Lambda ; r_{0}\right)$. There exists $0<h_{1} \leqq h_{0}$ such that $U_{0}$ is positively invariant under (1.2) for all $h<h_{1}$, that is

$$
x_{n}(h) \in U_{0} \quad \text { for } n=1,2,3, \cdots
$$

whenever $x_{0} \in U_{0}$.

Proof. The set $U_{0}$ is open because the Lyapunov function $V$ is continuous and contains $\Lambda$ because this is the set of points at which $V$ vanishes. Moreover for any $x \in U_{0}$

$$
\alpha(\operatorname{dist}(x, \Lambda)) \leqq V(x)<\alpha\left(r_{0}\right),
$$

so dist $(x, \Lambda)<r_{0}$, that is $x \in S\left(\Lambda ; r_{0}\right)$.

We define $h_{1}$ to be the largest $h \leqq h_{0}$ such that

$$
\frac{L C_{p} h^{p+1}}{1-e^{-c h}}<\frac{1}{4} \alpha\left(\frac{1}{2} \beta^{-1}\left(\alpha\left(r_{0}\right)\right)\right)^{2}
$$

for each $0<h<h_{1}$. Let $x_{0} \in U_{0}$ and $h<h_{1}$. By Lemma 3.1 we find $x_{1}(h) \in S\left(\Lambda ; R_{0}\right)$, the domain of definition of $V$. Hence inequality (3.3) is valid and gives

$$
V\left(x_{1}(h)\right) \leqq e^{-c h} \alpha\left(r_{0}\right)+L C_{p} h^{p+1}<\alpha\left(r_{0}\right),
$$

as $\alpha\left(\frac{1}{2} \beta^{-1}\left(\alpha\left(r_{0}\right)\right)\right)<\alpha\left(r_{0}\right)$, so that $x_{1}(h) \in U_{0}$. The proof is then completed by induction.

\footnotetext{
${ }^{2}$ The reason for this seemingly mysterious bound will be revealed in Lemma 3.6.
} 
Let $A$ and $B$ be nonempty, compact subsets of $\Re^{N}$. We define the Hausdorff separation of $\boldsymbol{A}$ from $\boldsymbol{B}$ by (see e.g. Roxin [7])

$$
H^{*}(A, B)=\max \{\operatorname{dist}(a, B) ; a \in A\}
$$

and the Hausdorff distance between $A$ and $B$ by

$$
H(A, B)=\max \left\{H^{*}(A, B), H^{*}(B, A)\right\} \text {. }
$$

( $H$ is a metric on the space of nonempty compact subsets of $\Re^{N}$.) In addition we define

$$
\eta(h)=\frac{2 L C_{p} h^{p+1}}{1-e^{-c h}}
$$

for $0<h<h_{1}$, so $0<\eta(h)<\frac{1}{2} \alpha\left(\frac{1}{2} \beta^{-1}\left(\alpha\left(r_{0}\right)\right)\right)$, and we set

$$
\Lambda(h)=\left\{x \in S\left(\Lambda ; R_{0}\right) ; V(x) \leqq \eta(h)\right\} .
$$

LEMMA 3.3. $\Lambda(h)$ contains $\Lambda$ in its interior and is compact for each $0<h<h_{1}$. Moreover

$$
H(\Lambda(h), \Lambda) \rightarrow 0 \quad \text { as } h \rightarrow 0^{+} .
$$

Proof. $\Lambda$ is the set of points on which the Lyapunov function $V$ vanishes, so $\Lambda$ is contained in $\Lambda(h)$. In fact it is contained in the interior of $\Lambda(h)$ since $V$ is continuous and $\eta(h)>0$. Moreover, $\Lambda(h)$ is bounded and closed, thus compact. Since $\Lambda \subset \Lambda(h)$, $H^{*}(\Lambda, \Lambda(h))=0$, so

$$
H(\Lambda(h), \Lambda)=H^{*}(\Lambda(h), \Lambda) \leqq \alpha^{-1}(\eta(h)) \rightarrow 0 \quad \text { as } h \rightarrow 0^{+} .
$$

Since $\eta(h)<\alpha\left(r_{0}\right)$ the set $\Lambda(h)$ is contained in the invariant set $U_{0}$ for $0<h<h_{1}$. We shall now show that $\Lambda(h)$ is uniformly asymptotically stable for the discrete dynamical system (1.2) for $0<h<h_{2}<h_{1}$ for some $h_{2}<h_{1}$.

LEMMA 3.4. $\Lambda(h)$ is positively invariant under (1.2) for each $0<h<h_{1}$.

Proof. Let $0<h<h_{1}$ and $x_{0} \in \Lambda(h)$. By Lemma 3.2 $x_{1}(h) \in U_{0}$ and inequality (3.3) holds, so

$$
\begin{aligned}
V\left(x_{1}(h)\right) & \leqq e^{-c h} V\left(x_{0}\right)+L C_{p} h^{p+1} \\
& \leqq e^{-c h} \eta(h)+\frac{1}{2} \eta(h)\left(1-e^{-c h}\right) \\
& =\frac{1}{2}\left(1+e^{-c h}\right) \eta(h)<\eta(h),
\end{aligned}
$$

where we have used definition (3.4) of $\eta(h)$. Hence $x_{1}(h) \in \Lambda(h)$. The proof is then completed by induction.

LEMMA 3.5. There exists an $0<h_{2} \leqq h_{1}$ such that

$$
V\left(x_{1}(h)\right) \leqq e^{-c h / 4} V\left(x_{0}\right)
$$

for any $x_{0} \in U_{0} \backslash \Lambda(h)$ and $0<h<h_{2}$.

Proof. By Lemma 3.2 we find $x_{1}(h) \in U_{0}$ and inequality (3.3) holds, so

$$
\begin{aligned}
V\left(x_{1}(h)\right) & \leqq e^{-c h} V\left(x_{0}\right)+L C_{p} h^{p+1} \\
& \leqq e^{-c h} V\left(x_{0}\right)+\frac{1}{2} \eta(h)\left(1-e^{-c h}\right) \\
& <\frac{1}{2}\left(1+e^{-c h}\right) V\left(x_{0}\right),
\end{aligned}
$$

for any $0<h<h_{1}$ since $V\left(x_{0}\right)>\eta(h)$. We define

$$
h_{2}=\min \left\{h_{1}, \gamma\right\}
$$


where $\gamma>0$ satisfies $e^{-c \gamma}+1=2 e^{-c \gamma / 4}$. Then

$$
\frac{1}{2}\left(1+e^{-c h}\right)<e^{-c h / 4}
$$

and hence

$$
V\left(x_{1}(h)\right)<e^{-c h / 4} V\left(x_{0}\right)
$$

for any $0<h<h_{2}$.

Repeating the above argument, we obtain

$$
V\left(x_{n}(h)\right) \leqq e^{-c n h / 4} V\left(x_{0}\right)
$$

if $x_{j}(h) \in U_{0} \backslash \Lambda(h)$ for $j=0,1, \cdots, n-1$. By the definition of $U_{0}$ we have $V\left(x_{0}\right)<\alpha\left(r_{0}\right)$, so

$$
V\left(x_{n}(h)\right) \leqq e^{-c n h / 4} \alpha\left(r_{0}\right)
$$

which cannot remain larger than $\eta(h)$ for all $n$. Hence there is a finite $n$ for which $V\left(x_{n}(h)\right) \leqq \eta(h)$, that is $x_{n}(h) \in \Lambda(h)$. Further iterates remain in $\Lambda(h)$ by Lemma 3.4. In fact, if $n h \geqq T(h)$ where

$$
T(h)=\frac{4}{c} \log \alpha\left(r_{0}\right)-\frac{4}{c} \log \eta(h),
$$

then $e^{-c n h / 4} \alpha\left(r_{0}\right) \leqq \eta(h)$, thus $x_{n}(h) \in \Lambda(h)$. Since $c h \geqq 1-e^{-c h}$, we have

$$
\eta(h)=\frac{2 L C_{p} h^{p+1}}{1-e^{-c h}} \geqq \frac{2 L C_{p}}{c} h^{p}
$$

and so

$$
T(h) \leqq T_{0}(h)=\frac{4}{c} \log \frac{c \alpha\left(r_{0}\right)}{L C_{p}}+\frac{4}{c} p \log \frac{1}{h} .
$$

LeMMA 3.6. There exists a $\delta_{0}>0$ such that

$$
x_{n}(h) \in \Lambda(h)
$$

for $n h \geqq T_{0}(h)$ and all $x_{0} \in S\left(\Lambda(h) ; \delta_{0}\right) \subset U_{0}$, provided $0<h<h_{2}$.

Proof. Let $\delta_{0}=\frac{1}{2} \beta^{-1}\left(\alpha\left(r_{0}\right)\right)>0$. For $x_{0} \in S\left(\Lambda(h), \delta_{0}\right)$ we have

$$
\begin{aligned}
\operatorname{dist}\left(x_{0}, \Lambda\right) & \leqq \operatorname{dist}\left(x_{0}, \Lambda(h)\right)+H(\Lambda(h), \Lambda) \\
& <\delta_{0}+\alpha^{-1}(\eta(h)) \\
& <\frac{1}{2} \beta^{-1}\left(\alpha\left(r_{0}\right)\right)+\frac{1}{2} \beta^{-1}\left(\alpha\left(r_{0}\right)\right) \\
& =\beta^{-1}\left(\alpha\left(r_{0}\right)\right) .
\end{aligned}
$$

Here we used $\eta(h)<\alpha\left(\frac{1}{2} \beta^{-1}\left(\alpha\left(r_{0}\right)\right)\right)$. We conclude

$$
V\left(x_{0}\right) \leqq \beta\left(\operatorname{dist}\left(x_{0}, \Lambda\right)\right)<\alpha\left(r_{0}\right),
$$

and so $x_{0} \in U_{0}$. The argument preceding the statement finishes the proof of the lemma.

The set $\Lambda(h)$ not only attracts iterates of the discretization (3.2) at a uniform rate, but in fact absorbs these iterates in a finite time no greater than $T_{0}(h)$ provided $0<h<h_{2}$. It remains now only to prove that $\Lambda(h)$ is uniformly stable for the discrete system (3.2). 
Lemma 3.7. Let $0<h<h_{2}$. For each $\varepsilon>0$ there exists a $\delta=\delta(\varepsilon, h)>0$ such that

$$
x_{n}(h) \in S(\Lambda(h) ; \varepsilon)
$$

for $n=1,2,3, \cdots$ and all $x_{0} \in S(\Lambda(h) ; \delta)$.

Proof. We define

$$
\delta=\delta(\varepsilon, h)=\min \left\{\delta_{0}, \frac{1}{2} \varepsilon\left(1+h L_{p}(h)\right)^{-T_{0}(h) / h}\right\},
$$

where $L_{p}(h)$ is the uniform Lipschitz constant for $F_{h}$. Consider any $x_{0} \in S(\Lambda(h) ; \delta)$. If $x_{0} \in \Lambda(h)$, then $x_{n}(h) \in \Lambda(h)$ for $n=1,2,3, \cdots$ since $\Lambda(h)$ is positively invariant under (1.2), so (3.5) is automatically satisfied. Consequently we suppose that $x_{0} \notin \Lambda(h)$. As $\Lambda(h)$ is compact there exists a $y_{0} \in \Lambda(h)$ such that

$$
\operatorname{dist}\left(x_{0}, \Lambda(h)\right)=\left|x_{0}-y_{0}\right| \text {. }
$$

Let $x_{n}(h)$ and $y_{n}(h)$ be the iterative sequences for (1.2) starting at $x_{0}$ and $y_{0}$, respectively. Then

$$
\begin{aligned}
\left|x_{n}(h)-y_{n}(h)\right| & \leqq\left|x_{n-1}(h)-y_{n-1}(h)\right|+h\left|F_{n}\left(x_{n-1}(h)\right)-F_{n}\left(y_{n-1}(h)\right)\right| \\
& \leqq\left(1+h L_{p}(h)\right)\left|x_{n-1}(h)-y_{n-1}(h)\right| \\
& \ldots \\
& \leqq\left(1+h L_{p}(h)\right)^{n}\left|x_{0}-y_{0}\right| .
\end{aligned}
$$

As $\Lambda(h)$ is positively invariant we find $y_{n}(h) \in \Lambda(h)$ for $n=1,2, \cdots$, so

$$
\begin{aligned}
\operatorname{dist}\left(x_{n}(h), \Lambda(h)\right) & \leqq\left|x_{n}(h)-y_{n}(h)\right| \\
& \leqq\left(1+h L_{p}(h)\right)^{n} \delta \\
& \leqq\left(1+h L_{p}(h)\right)^{T_{0}(h) / h} \delta<\varepsilon
\end{aligned}
$$

for $n h<T_{0}(h)$, by the choice of $\delta$. In addition we have $x_{0} \in S\left(\Lambda(h) ; \delta_{0}\right)$, so by Lemma 3.6

$$
x_{n}(h) \in \Lambda(h) \text { for } n h \geqq T_{0}(h) .
$$

Hence the inclusion (3.5) holds for all $n=1,2,3, \cdots$, so $\Lambda(h)$ is uniformly stable for (3.2).

In summary, the compact set $\Lambda(h)$ is uniformly asymptotically stable for the discrete dynamical system (1.2) for all step-sizes $0<h<h_{2}$. It contains $\Lambda$ in its interior and converges to $\Lambda$ with respect to the Hausdorff metric as $h \rightarrow 0^{+}$. In fact, it is more than just attracting as required by the definition of uniform asymptotic stability; it actually absorbs all neighboring iterates in a finite time $T_{0}(h)$.

This complete the proof of Theorem 1.1.

Remarks. 1. As $h$ tends to zero the time $T_{0}(h)$ tends to infinity. This is reasonable since $\Lambda(h)$ approaches the (continuous time) attracting set $\Lambda$ and $\Lambda$ does not necessarily absorb nearby trajectories in finite time.

2. The factor $4 p / c$ in front of the term $\log 1 / h$ in $T_{0}(h)$ can be improved: For any $0<\tilde{c}<c$ the factor can be reduced to $p / \tilde{c}$. Only slight modifications of our arguments are necessary to see this.

3. From the proof of Lemma 3.3 it is clear that the convergence rate of $\Lambda(h)$ to $\Lambda$ in the Hausdorff metric is $O\left(\alpha^{-1}\left(h^{p}\right)\right)$. For point and limit cycle attractors the 
Lyapunov function can often by chosen such that $\alpha^{-1}(x)=O(x)$, in which case $\Lambda(h)$ converges to $\Lambda$ at the rate $O\left(h^{p}\right)$. See for example [9], [10].

4. Additional remarks. We have assumed nothing about the dynamics of the continuous system (1.1) within its attracting set $\Lambda$, and so have been able to say nothing about the dynamics of the discretized systems (1.2) within their attracting sets $\Lambda(h)$, apart from the positive invariance established in Lemma 3.4. Our choice of the terminology "attracting set" rather than "attractor" reflects this omission, with the latter term being reserved to mean an attracting set which contains a dense trajectory.

With additional assumptions about the attracting sets $\Lambda$, we expect to be able to say more about the sets $\Lambda(h)$ and their internal dynamics. For example, if $\Lambda=\{\bar{x}\}$ is a singleton set, the sets $\Lambda(h)$ are small neighborhoods of this point and contain a unique point $\bar{x}(h)$ which is asymptotically stable for the discretized system (1.2). Similarly, when $\Lambda$ is a closed curve representing a periodic solution of the continuous system (1.1), in which case uniform asymptotic stability coincides with orbital stability, then the sets $\Lambda(h)$ will be thin tubes about this curve, possibly containing closed curves which are invariant and asymptotically stable for the discrete system (1.2). See for example [3] for results in this direction. In general, if $\Lambda$ contains a dense trajectory of system (1.1), we expect that $\Lambda(h)$ may contain a trajectory of (1.2) which is "pseudo-dense" in $\Lambda$, that is, which returns infinitely often to a ball of some radius $r(h)$ around any point of $\Lambda$, where $r(h) \rightarrow 0$ and $h \rightarrow 0$. Such a trajectory would sweep out, approximately, the shape of $\Lambda$, although it may not closely shadow a particular trajectory of the continuous system. We hope to develop and provide proofs of these speculations in a later paper.

It is clear that our construction of the attracting sets $\Lambda(h)$ is not unique, although for each $h$ the actual limit sets within the possible choices will be unique. In addition our construction and proofs in the previous section can be straightforwardly modified to handle one-step methods with variable step-sizes. The generalization to multi-step methods does not at the time of writing appear obvious to us.

A problem of practical significance is how to determine the stability characteristics of the attracting set $\Lambda$ of the original continuous system (1.1). Except in simple cases, it may be extremely difficult to explicitly determine an appropriate Lyapunov function, even though its existence be guaranteed by Theorem 2.1. This of course assumes that we know that $\Lambda$ is a uniformly asymptotically stable set for (1.1). In practice we usually only have an indication of this from numerical calculations. Then we may ask for a converse of Theorem 1.1, which appears possible but would lead to a coupling between the step-size and the stability characteristics of the attracting set of (1.2) for that step-size. A uniformity in these stability characteristics with decreasing step-size would be a strong indication for similar stability characteristics of the continuous system (1.1).

\section{REFERENCES}

[1] W.-J. BEYN, On discretization of bifurcation problems, in Bifurcation Problems and their Numerical Treatment, H. D. Mittelman and H. Weber, eds., Birkhäuser-Verlag, Basel, 1980.

[2] W.-J. BEYN AND E. J. DOEDEL, Stability and multiplicity of solutions to discretizations of nonlinear ordinary differential equations, SIAM J. Sci. Stat. Comp., 2 (1981), pp. 107-120.

[3] F. BREZZI, S. USHIKI AND H. FUJI, Real and ghost bifurcation dynamics in difference schemes for ODEs, in Numerical Methods for Bifurcation Problems, T. Küpper, H. D. Mittelmann and H. Weber, eds., Birkhäuser-Verlag, Basel, 1984.

[4] H. B. Keller, Approximation methods for nonlinear problems with application to two point boundary value problems, Math. Comp., 29 (1975), pp. 464-474. 
[5] H.-O. KREISS, Difference approximations for boundary and eigenvalue problems for ordinary differential equations, Math. Comp., 26 (1972), pp. 605-624.

[6] E. N. LoRenZ, Deterministic non-periodic flows, J. Atmospheric Sci., 20 (1963), pp. 130-141.

[7] E. O. Roxin, General control systems, J. Differential Equations, 1 (1965), pp. 115-150.

[8] C. SPARrow, The Lorenz Equations: Bifurcations, Chaos and Strange Attractors, Springer-Verlag, 1982.

[9] H. J. STETTER, Analysis of Discretization Methods for Ordinary Differential Equations, Springer-Verlag, 1973.

[10] T. YosHIZAWA, Stability theory by Lyapunov's second method, The Mathematical Society of Japan, 1966. 